\title{
OPTIMAL PRICING AND DETERIORATING INVENTORY CONTROL WHEN INVENTORY STIMULATES STOCHASTIC DEMAND WITH REFERENCE PRICE EFFECT
}

\author{
YONGRUI DUAN ${ }^{1,2}$ AND Yu CAO ${ }^{1, *}$
}

\begin{abstract}
We study the joint dynamic pricing and deteriorating inventory management problem in the presence of reference price and stock display effects. In a random potential market setting with convex replenishment/ordering and holding/shortage cost functions, the retailer replenishes/orders and sells a single deteriorating product over a continuous-time infinite horizon to maximize its profit. The demand rate depends negatively on the sales price and positively on the consumers' reference price and displayed stock quantity. The inventories deteriorate physically at a constant fraction of the on-hand stocks. A stochastic optimal control model is established to characterize the optimal policy in a linear feedback form of the state variables when the observed inventory level is either positive or negative. We also investigate the asymptotic behavior of the system and provide sufficient conditions for the stability and monotone convergence of the expected long-run behavior. Finally, we perform numerical examples to illustrate the theoretical results and sensitivity analysis to derive insights into deteriorating inventory management under reference price and stock display effects. The findings suggest that current reference price level has a positive effect while inventory level has a negative on the optimal replenishment rate and price. We characterize the sample paths of optimal strategies and find that the initial consumer reference price has an important impact on the firm's optimal operations management. On the expected longrun behavior, it is beneficial for the firm to reduce demand uncertainty and deteriorating rate. We also observe that a large factor of displayed stock effect brings a positive effect on the total expected profit. Additionally, the firm needs to reduce (increase) the price and increase (reduce) the replenishment rate with high reference price effect intensity (memory factor).
\end{abstract}

Mathematics Subject Classification. MSC2010:90B05, 90B30, 90B60, 90C39, 91E99, $93 \mathrm{E} 20$.

Received June 6, 2019. Accepted May 19, 2020.

\section{INTRODUCTION}

Consumers form a memory of past prices of items in their frequent transactions and past shopping experiences. They develop price expectations when consumers are exposed to sales prices for some time. This expectation, as a benchmark point against which the current price of products is compared, is characterized by the concept

Keywords. Deteriorating inventory, reference price- and inventory-dependent demand, dynamic replenishment and pricing, stochastic process, closed-loop policy.

1 School of Economics and Management, Tongji University, Shanghai 200092, P.R. China.

2 Bosch-Tongji University Chair of Global Supply Chain Management, Tongji University, Shanghai 200092, P.R. China.

* Corresponding author: caoyu0601@163.com 
of reference price [24,42]. The empirical evidence that consumers' reference price influences their purchasing behavior has been studied in the marketing literature $[30,40]$. The difference or relative difference between the reference price and the sales price plays an important role in product demand. If the current price exceeds the reference price, consumers perceive a loss or a surcharge and are less attracted to make the purchase, thus, demand decreases in the short term; otherwise, consumers perceive a gain or a discount which will lead to increased demand. This phenomenon is known as the reference price effect [10]. Consumers are loss-averse (lossseeking) if demand is more sensitive to gains (losses) than losses (gains); otherwise they are loss-neutral. The firms cannot directly affect how consumers form reference price but can influence it using a dynamic pricing strategy (e.g., skimming, penetration or cyclical) by considering consumers' reference price effect [26]. Dynamic pricing under reference price effect has attracted many academicians' attentions in the marketing and economics literature. For a review of relevant literature, see $[4,40]$.

However, most existing models in these streams of literature ignore the demand-stimulating role of retail inventories. The fact that displayed stocks have a stimulating effect on demand has been observed by marketing researchers and practitioners. In some retailing industries, it is expected that the demand rate depends on the displayed stock level. Especially for deteriorating items ${ }^{3}$, more items in shelf space attracts more consumers to purchase them [37]. This happens because of visibility and popularity which stimulates demand by creating novelty or impulse-buy opportunities. Consequently, the stock level built up often has a positive effect on the sales and the profitability. Wolfe [54] provides empirical evidence to support that the demand rate changes with the displayed inventory. Levin et al. [35] observe that large piles of consumer goods displayed in a supermarket will lead customers to buy more. Later, many researchers have developed inventory models to quantify this phenomenon. For example, Baker and Urban [5] first develop an EOQ model for a power-form inventory-leveldependent demand pattern. Mandal and Phaujdar [39] propose an economic production quantity model for deteriorating items with a linear stock-dependent demand rate. Pal et al. [44] generalize the inventory model of Baker and Urban [5] for deteriorating items with a constant deterioration rate. Certainly, a large number of other researchers have been focusing on deteriorating inventory model with an inventory-dependent demand rate, such as $[7,25,34,45,48,53]$ and so on.

Along with dynamic pricing, firms also focus on effective inventory schedules (e.g., replenishment or production strategies) in the presence of demand uncertainty and violent market competition. Lack of effective inventory management can significantly affect the pricing strategies and thus damage the profit of deteriorating products of a retailer. Although the importance of reference price and stock display effects has been repeatedly emphasized by both researchers and marketers, very few of them pay attention to these two effects simultaneously. In this paper, we investigate the dynamic pricing and deteriorating inventory strategy in the presence of reference price effect when displayed stock stimulates demand. In the continuous-time framework, we develop a single-item joint replenishment/ordering and pricing model of deteriorating inventory, incorporated with stochastic reference- and inventory-dependent demand rate. The reference price is modeled as a continuous weighted average of past prices with an exponentially decaying weighting function. The random disturbance of market potential is characterized as a stochastic differential equation which transforms the optimization problem into a stochastic control problem. We assume a constant proportion of the items on the shelf will deteriorate per time-unit and the unsatisfied demand is fully backlogged. We characterize the optimal feedback replenishment/ordering and pricing strategies of a profit-maximizing retailer when inventory level is either positive or negative. Moreover, we derive the expected steady-states and provide sufficient conditions for their stability and monotone convergence. Finally, a numerical example is presented to illustrate the developed model. We also carry out the sensitivity analysis of the optimal solutions with respect to the main model parameters. Our analysis highlights the impact of reference price and stock displayed effects on the firm's optimal strategies and profitability.

\footnotetext{
${ }^{3}$ According to Li et al. [37], the deterioration is defined as the damage, spoilage, dryness, vaporization, etc., that results in the decreasing usefulness from the original condition. Examples include almost all food stuffs, fashionable items, electronic products, etc.
} 
In particular, we make the following contributions. First, to the best of our knowledge, this study is the first paper to integrate reference price and stock displayed effects into stochastic replenishment inventory model for deteriorating items in a continuous-time framework. Second, this work proposes a different approach, i.e., stochastic optimal control, to modeling and analyzing the above problem. This approach has been proved efficiently in many economic, managerial and industrial applications (see, e.g., [49]). By employing the dynamic programming principle, the stochastic control model is transformed into a Hamilton-Jacobi-Bellman (HJB) equation which characterizes the optimal solutions in a linear feedback form with respect to the two state variables. The advantage of our continuous approach avoids the curse of dimensionality and thus requires computation efforts to solve the HJB equation.

The rest of this paper is organized as follows. Section 2 reviews the literature related to this study. We describe the basic notation, assumptions, and formulation of our model in Section 3. Section 4 provides the model analysis. Section 5 conducts a numerical example and presents the sensitivity analysis to demonstrate the theoretical results. Section 6 is for conclusion and future research. All proofs are relegated to the appendix.

\section{Literature REVIEW}

In this section, we review the literature related to this study and identify the research gap to clarify our contribution. This work is built upon three streams of research in the literature: (a) reference price effect, (b) deteriorating inventory management with stock displayed effect, and (c) joint replenishment/production and pricing strategy.

The topic of reference price has been studied in economics and marketing literature. Sorger [50] and Kopalle and Winer [33] model the reference price as a continuous weighted average of the historical price exposures of the consumer with an exponentially decaying weighting function. Kalyanaram and Winer [30] first empirically support that consumers use reference prices in brand choices and they rely on past prices as part of the reference price formation process. Briesch et al. [6] empirically evaluate five alternative reference price models. They find that the model based on the brand's own price history, i.e., a memory-based model, is the best. In a continuoustime framework, Fibich et al. [22] propose a two-stage optimization method to solve the nonsmooth dynamic pricing problem under asymmetric reference price effect and deterministic linear demand. Popescu and Wu [47] generalize their work under more general demand with reference effect in a discrete-time setting. Zhang et al. [57] extend the work of Fibich et al. [22] to a competitive supply chain. Chenavaz [11] investigates the structural properties of a general demand function under reference price effect with an optimal control framework. The author shows that the reference price effect weakens the market power of the firm and constitutes a main driver of the dynamics of the selling price. Hu et al. [9] investigate a finite-horizon dynamic pricing model under asymmetric reference effect. They identify several structural properties of the problem and develop strongly polynomial-time algorithms to compute the optimal strategies for some plausible scenarios. Interested readers are referred to [4] for other articles on dynamic pricing models with reference price effect consideration. However, the above papers do not consider the replenishment/inventory decision and ignore the deterioration of items. Different from these studies, we explicitly model the deteriorating inventory in our setting and consider stock displayed effect.

The literature on the EOQ inventory model for deteriorating items under inventory-dependent demand is rich. Padmanabhan and Vrat [43] develop a deteriorating EOQ inventory model with a stock dependent selling rate and a constant deterioration rate. They solve the EOQ model in cases of complete, partial, and no backlogging, respectively. Dye and Ouyang [15] extend the work of Padmanabhan and Vrat [43] by considering a timeproportional backlogging rate and show the existence and uniqueness of the solutions. Yang et al. [56] investigate a deteriorating inventory under inflation and stock-dependent consumption rate in the presence of partial backlogging, time-varying replenishment cycles, and time-varying shortage intervals. Lee and Dye [34] establish a deteriorating inventory model with stock-dependent demand. They optimize joint preservation technology cost and replenishment schedule to maximize the total profit per unit time and provide a simple algorithm to identify the optimal solution. Recently, a large body of papers in this stream of literature also integrate 
price factor. For example, an EPQ model for deteriorating items is formulated by Teng and Chang [52] when the demand function is price- and inventory-display-dependent. They assume the limited shelf space and give the necessary conditions to obtain an optimal solution. Hou and Lin [28] integrate inflation and time value of money into a deterministic EOQ deteriorating inventory model with price- and inventory-dependent selling rates. Mishra et al. [41] address an EOQ inventory problem for deteriorating seasonal items by incorporating price- and stock-dependent demand function and preservation technology investment in the case of complete or partial backordering. Feng et al. [20] derive the optimal selling price, cycle time, and non-zero ending inventory level when the demand rate for perishable products depends on selling price, freshness and displayed stocks. They show that the profit is strictly pseudo-concave in the decision variables. Our paper differs from the above studies by integrating dynamic pricing and reference price effect into a stochastic inventory management model for deteriorating items when displayed stock has a positive effect on demand rate.

There is an extensive literature on joint dynamic replenishment/production and pricing decisions using control theory in continuous-time framework ${ }^{4}$. Pekelman [46] first use control theory to study a continuous-time price and production schedule over a finite horizon with time-dependent demand rate and nonnegative inventory constraint. Thereafter, many researchers extend this seminal work under different assumptions, including nonlinear demand function [18]; cost and demand learning effects [29]; no backorders [1]; competition [2]; distribution channel [16,17]; demand uncertainty [27] and so on. Some papers in this stream of literature also integrate deterioration of products. Feng et al. [21] extend the work of Pekelman [46] into perishable items with a constant deterioration rate. Feng [19] furthers extend their work to dynamic pricing, quality investment and production/replenishment problem for perishable items. Lu et al. [38] investigate joint pricing and replenishment inventory system for perishable items when the deterministic demand rate depends both on selling price and inventory level displayed. Xue et al. [55] obtain the optimal dynamic pricing strategies for deteriorating items when demand is deterministic and reference-price dependent. Li et al. [38] and Duan et al. [14] consider a joint dynamic production and pricing deteriorating inventory model under stochastic setting over an infinite horizon and a finite horizon, respectively. In contrast to the above papers, we integrate reference price and stock display effects into the deteriorating inventory model under stochastic demand in a continuous-time framework.

In summary, to highlight the contributions of this work, Table 1 summarizes the major model settings of this paper and the relevant literature.

\section{MODEL DESCRIPTION}

\subsection{Basic notation and assumptions}

For ease of reference, the basic notation to be used in the following joint replenishment/ordering and pricing inventory model is summarized in Table 2. Also, we need the following assumptions:

- A retailer maintains an inventory system of a single deteriorating product to meet consumers' demand.

- The retailer dynamically replenishes/orders and sells the items over an infinite horizon to maximize his expected profit.

- The replenishment rate is infinite and the lead time is zero.

- The shortage is allowed.

Reference price formulation. Following [22,33,50], we model the reference price $r(t)$ as an exponential smoothing process of historical price representing a decaying memory effect as follows:

$$
r(t)=\beta e^{-\beta t} \int_{-\infty}^{t} e^{\beta s} p(s) \mathrm{d} s
$$

\footnotetext{
${ }^{4}$ We refer the interested readers to [8] for a comprehensive review on joint pricing and inventory management in a discrete-time framework.
} 
TABLE 1. Summary of the relevant research.

\begin{tabular}{|c|c|c|c|c|c|c|}
\hline References & $\begin{array}{l}\text { Deteriorating } \\
\text { items }\end{array}$ & $\begin{array}{l}\text { Deterministic/ } \\
\text { stochastic } \\
\text { demand }\end{array}$ & $\begin{array}{l}\text { Price- } \\
\text { dependent } \\
\text { demand }\end{array}$ & $\begin{array}{l}\text { Reference } \\
\text { price- } \\
\text { dependent } \\
\text { demand }\end{array}$ & $\begin{array}{l}\text { Inventory- } \\
\text { dependent } \\
\text { demand }\end{array}$ & $\begin{array}{l}\text { Dynamic/ } \\
\text { static } \\
\text { decisions }\end{array}$ \\
\hline Sorger $[50]$ & No & Deterministic & Yes & Yes & No & Dynamic \\
\hline Fibich et al. [22] & No & Deterministic & Yes & Yes & No & Dynamic \\
\hline Popescu and $\mathrm{Wu}[47]$ & No & Deterministic & Yes & Yes & No & Dynamic \\
\hline Chenavaz [11] & No & Deterministic & Yes & Yes & No & Dynamic \\
\hline $\mathrm{Hu}$ et al. $[9]$ & No & Deterministic & Yes & Yes & No & Dynamic \\
\hline Padmanabhan and Vrat [43] & Yes & Deterministic & No & No & Yes & Static \\
\hline Dye and Ouyang [15] & Yes & Deterministic & No & No & Yes & Static \\
\hline Lee and Dye [34] & Yes & Deterministic & No & No & Yes & Static \\
\hline Teng and Chang [52] & Yes & Deterministic & Yes & No & Yes & Static \\
\hline Mishra et al. [41] & Yes & Deterministic & Yes & No & Yes & Static \\
\hline Pekelman $[46]$ & No & Deterministic & Yes & No & No & Dynamic \\
\hline Herbon and Kogan [27] & No & Stochastic & Yes & No & No & Dynamic \\
\hline Feng et al. [21] & Yes & Deterministic & Yes & No & No & Dynamic \\
\hline Feng $[19]$ & Yes & Deterministic & Yes & No & No & Dynamic \\
\hline Lu et al. [38] & Yes & Deterministic & Yes & No & Yes & Dynamic \\
\hline Xue et al. [55] & Yes & Deterministic & Yes & Yes & No & Dynamic \\
\hline Li et al. [38] & Yes & Deterministic & Yes & No & No & Dynamic \\
\hline Duan et al. [14] & Yes & Stochastic & Yes & No & No & Dynamic \\
\hline Present paper & Yes & Stochastic & Yes & Yes & Yes & Dynamic \\
\hline
\end{tabular}

where $\beta$ is the memory parameter or a proxy of customer loyalty [47] and $p(t)$ is the selling price at time $t$. Differentiating the above equation, we obtain the following ordinary differential equation:

$$
\mathrm{d} r(t)=\beta(p(t)-r(t)) \mathrm{d} t, \quad r(0)=r_{0},
$$

where $r_{0}$ is the initial reference price at time $t=0$ and can be determined by, for example, prices of similar products.

Demand function. Assume that the demand function is dependent on the reference price and displayed stock. Let $x(t)$ be the stock level on the shelf. The reference price- and inventory-dependent demand rate $D(p(t), r(t), x(t))$ builds upon a standard linear demand function $D(p(t))=a-b p(t)$ (see, e.g., $[12,16]$ ) in the absence of reference and stocks display effects (i.e., $\gamma=\alpha=0)$. More specifically, it is specified as follows:

$$
D(p(t), r(t), x(t))=a-b p(t)+\gamma(r(t)-p(t))+\alpha x(t) \mathbb{1}_{x(t) \geq 0},
$$

where $a, b, \gamma$ and $\alpha$ are nonnegative parameters. $b$ captures the impact of the price on demand. $\mathbb{1}_{A}=1$ is the indicator function of $A: \mathbb{1}_{A}(x)=1$, if $x \in A$, and 0 otherwise. Hence, $x(t) \mathbb{1}_{x(t) \geq 0}$ is the stock on display at time $t$. Our demand function specifies that, first, the market potential is the sum of a constant $a$, typically in the classical linear demand model, and a time-varying function $\alpha x(t) \mathbb{1}_{x(t) \geq 0}$, where $\alpha$ is the sensitivity of the demand with respect to the displayed stock level. As a result, a higher display stock level induces a larger market potential. The linear stock-dependent demand rate is common in the literature, e.g., [39]. Second, the demand rate negatively depends on product price, capturing the economic role of price, a standard assumption. Finally, the term $\gamma(r(t)-p(t))$ indicates the consumers' reference price effect, where $\gamma$ measures the intensity of this effect. We assume that the demand is in the form of linear and additive reference price effect (see, e.g., [22]). Consumers perceive gains if the selling price is below the reference price; otherwise losses. Further, we assume the reference price effect is symmetric, i.e., consumers are loss/gain neutral. 
TABLE 2. The notation used throughout this paper.

\begin{tabular}{ll}
\hline \hline Parameters & \\
$t$ & Continuous-time argument, $t \in[0, \infty)$ \\
$a$ & Mean market size, $a<\infty$ \\
$b$ & Demand sensitivity to the price, $b>0$ \\
$D(p(t), r(t), x(t))$ & Demand rate function at time $t$ \\
$Q(t)$ & Cumulative demand up to time $t$ \\
$\gamma$ & Reference price effect intensity \\
$\beta$ & Memory parameter or the speed of adjustment of the reference price \\
$\alpha$ & Sensitivity of the demand to the displayed stock level \\
$\theta$ & Constant deterioration coefficient \\
$W(t)$ & Standard Wiener process $($ Brownian motion) \\
$\sigma$ & Volatility parameter of the market size \\
$x_{0}, r_{0}$ & Initial inventory level and reference level, respectively \\
$h, s$ & Inventory/shortage cost coefficient, $h, s>0$ \\
$c$ & Replenishment cost coefficient, $c>0$ \\
$E$ & Expectation operator \\
$p, \bar{p}$ & The lower bound and upper bound of the price, respectively, $\bar{p}>\underline{p}>0$ \\
$\rho$ & Discount factor \\
$\mathcal{A}$ & Admissible control set \\
$V(x, r)$ & Profit-to-go function \\
$C$ control variables & \\
$p(t)$ & Product price at time $t, p(t) \in[p, \bar{p}]$ \\
$u(t)$ & Replenishment rate at time $t, u(t) \in[0, \infty)$ \\
$S t a t e$ variables & \\
$x(t)$ & Inventory level at time $t$ \\
$r(t)$ & Reference price level at time $t$ \\
\hline &
\end{tabular}

Furthermore, let $Q(t)$ denote the cumulative demand up to time $t$, and it satisfies the following stochastic process:

$$
\mathrm{d} Q(t)=D(p(t), r(t), x(t)) \mathrm{d} t-\sigma \mathrm{d} W(t), \quad t \geq 0,
$$

where $\sigma^{2}$ is the variance capturing a random shock on the market potential and $W(t)$ denotes a standard Weiner process.

Inventory dynamics. The retailer displays all the products ordered on the ample shelf to stimulate demand. Let $u(t)$ be the instantaneous replenishment rate. Then the inventory level $x(t)$ satisfies the following continuoustime dynamics captured by the Itó stochastic differential equation:

$$
\mathrm{d} x(t)=u(t) \mathrm{d} t-f(x(t)) \mathbb{1}_{x(t) \geq 0}-\mathrm{d} Q(t) .
$$

Furthermore, considering equations (3.3) and (3.2), the above inventory dynamics becomes:

$\mathrm{d} x(t)=\left(u(t)-f(x(t)) \mathbb{1}_{x(t) \geq 0}-\left(a-b p(t)+\gamma(r(t)-p(t))+\alpha x(t) \mathbb{1}_{x(t) \geq 0}\right)\right) \mathrm{d} t+\sigma \mathrm{d} W(t), \quad x(0)=x_{0}, \quad t \geq 0$,

where $x_{0}$ is the initial inventory stock. Assume that items deteriorate immediately once they are placed on the retail shelf. For analytical tractability and model clarification, we assume $f(x(t))=\theta x(t)$, i.e., the stocks deteriorate physically at a constant fraction $\theta$ of the on-hand inventory, this assumption has been commonly used in the deteriorating inventory literature [19, 21, 38, 43, 44]. Equation (3.4) implies that the instantaneous change of the display stock level is stochastic whereas the current stock level is known. This random inventory system comprises two components. The deterministic component $\left(u(t)-f(x(t)) \mathbb{1}_{x(t) \geq 0}-\right.$ 
$\left(a-b p(t)+\gamma(r(t)-p(t))+\alpha x(t) \mathbb{1}_{x(t) \geq 0}\right)$ in equation (3.4), i.e., the drift coefficient, represents the expected change in stock level at time $t$ under replenishment rate $u(t)$ and sales price $p(t)$. The random component of (3.4) involves the stochastic demand process $\sigma \mathrm{d} W(t)$, where $\sigma$ is referred to as the diffusion coefficient. If the difference between the accumulated quantity ordered and demand at time $t$ is positive (i.e., the stock surplus $x(t)>0$ ), the display products deteriorate at a given rate and are used to satisfy consumers' demand. As a consequence, an inventory holding cost is incurred. Otherwise, a shortage (i.e., $x(t)<0)$ occurs and orders are fully backlogged which results in a backlog cost. In line with many replenishment/production control models (see, e.g., $[2,14,17,38]$ ), we assume that the holding/shortage costs and replenishment cost to be convex and quadratic as follows

$$
H(x(t))=\left\{\begin{array}{ll}
h x^{2}(t), & x(t) \geq 0, \\
s x^{2}(t), & x(t)<0,
\end{array} \text { and } \quad C(u(t))=c u^{2}(t),\right.
$$

where the holding/shortage cost coefficients $h$ and $s$, as well as replenishment cost parameter $c$ are positive.

\subsection{Joint replenishment and pricing inventory model}

Let $\mathbb{E}[\cdot]$ denote the expectation operation, then the retailer's objective functional is specified as:

$$
\begin{aligned}
\mathbb{E} \int_{0}^{\infty} & e^{-\rho t}[p(t) \mathrm{d} Q(t)-(C(u(t))+H(x(t)))] \mathrm{d} t \\
= & \mathbb{E} \int_{0}^{\infty} e^{-\rho t}[p(t) D(p(t), r(t), x(t))-C(u(t))-H(x(t))] \mathrm{d} t-\mathbb{E} \int_{0}^{\infty} e^{-\rho t} p(t) \sigma \mathrm{d} W(t) \\
\quad= & \mathbb{E} \int_{0}^{\infty} e^{-\rho t}\left[p(t)\left(a-b p(t)+\gamma(r(t)-p(t))+\alpha x(t) \mathbb{1}_{x(t) \geq 0}\right)-C(u(t))-H(x(t))\right] \mathrm{d} t,
\end{aligned}
$$

where $\rho>0$ is the discount factor. The first equality holds due to equation (3.3) and the last equality is derived by equation (3.2) and $\mathbb{E} \int_{0}^{\infty} e^{-\rho t} p(t) \sigma \mathrm{d} W(t)=0$ by Theorem 4.4.14(e) in [3].

We model the retailer's operations as a continuous-time stochastic joint pricing and deteriorating inventory control problem. Let $\mathcal{A}=\{(u(t), p(t)) \mid(u(t), p(t)) \in[0, \infty) \times[\underline{p}, \bar{p}]\}$ denote the retailer's admissible control set. The goal is to find the optimal policy $\left(u^{*}(t), p^{*}(t)\right) \in \mathcal{A}$ to maximize the firm's expected intertemporal profit over the time horizon $[0, \infty)$ subject to the state equations (3.1) and (3.4), that is,

$$
\max _{(u(t), p(t)) \in \mathcal{A}} E \int_{0}^{\infty} e^{-\rho t}\left[p(t)\left(a-b p(t)+\gamma(r(t)-p(t))+\alpha x(t) \mathbb{1}_{x(t) \geq 0}\right)-C(u(t))-H(x(t))\right] \mathrm{d} t
$$

subject to

$$
\begin{aligned}
\mathrm{d} x(t) & =\left(u(t)-a+(b+\gamma) p(t)-\gamma r(t)-(\theta+\alpha) x(t) \mathbb{1}_{x(t) \geq 0}\right) \mathrm{d} t+\sigma \mathrm{d} W(t), \quad x(0)=x_{0}, \\
\mathrm{~d} r(t) & =\beta(p(t)-r(t)) \mathrm{d} t, \quad r(0)=r_{0}, \quad t \geq 0,
\end{aligned}
$$

where $x(t)$ and $r(t)$ are the state variables and $u(t)$ and $p(t)$ are the control variables. Hereafter, we omit the time argument when no ambiguity may arise.

\section{Optimal Decisions}

We will solve model (3.5) via the development of the Hamilton-Jacobi-Bellman (HBJ for short) equation satisfied by a certain "value function". To this end, a standard stochastic dynamic programming technique will be adopted. More specifically, we define $V(x, r)$ as the profit-to-go function, i.e., the optimal profit onward if the current inventory and reference price pair is $(x, r)$. Formally, $V(x, r)$ can be defined as

$$
V(x, r)=\max _{(u, p) \in \mathcal{A}} E \int_{t}^{\infty} e^{-\rho t}\left[p\left(a-b p+\gamma(r-p)+\alpha x \mathbb{1}_{x(t) \geq 0}\right)-C(u)-H(x)\right] \mathrm{d} t
$$


The principle of optimality gives

$$
V(x, r)=\max _{(u, p) \in \mathcal{A}} E\left\{\left[p\left(a-b p+\gamma(r-p)+\alpha x \mathbb{1}_{x(t) \geq 0}\right)-C(u)-H(x)\right] \mathrm{d} t+V(x+\mathrm{d} x, r+\mathrm{d} r)\right\},
$$

By Taylor's expansion and the theory of stochastic calculus [3], the corresponding HJB equation reads as

$$
\begin{aligned}
\rho V= & \max _{(u, p)}\left\{p\left(a-b p+\gamma(r-p)+\alpha x \mathbb{1}_{x(t) \geq 0}\right)-C(u)-H(x)+V_{x}(u-a+(b+\gamma) p\right. \\
& \left.\left.-\gamma r-(\theta+\alpha) x \mathbb{1}_{x \geq 0}\right)+V_{r} \beta(p-r)+\frac{1}{2} \sigma^{2} V_{x x}\right\},
\end{aligned}
$$

where $V_{i}=\partial V / \partial i$ and $V_{i i}=\partial^{2} V / \partial i^{2}, i=x, r$. The marginal valuations $V_{x}$ and $V_{r}$ measure the instantaneous change in $V(x, r)$ per unit of the inventory and reference price, i.e., the shadow price.

The first-order optimality conditions imply

$$
\begin{aligned}
& u^{*}=\frac{V_{x}}{2 c}, \\
& p^{*}=\frac{a+\gamma r+\alpha x \mathbb{1}_{x \geq 0}}{2(b+\gamma)}+\frac{V_{x}}{2}+\frac{\beta V_{r}}{2(b+\gamma)} .
\end{aligned}
$$

Next, inserting (4.3) and (4.4) into the right-hand side of the HJB equation (4.2) yields

$$
\begin{aligned}
\rho V= & \left(\frac{1}{4 c}+\frac{b+\gamma}{4}\right) V_{x}^{2}+\frac{\beta^{2}}{4(b+\gamma)} V_{r}^{2}+\frac{\beta}{2} V_{x} V_{r}-\frac{a+\gamma r+(\alpha+2 \theta) x \mathbb{1}_{x \geq 0}}{2} V_{x} \\
& +\left(\frac{\beta\left(a+\gamma r+\alpha x \mathbb{1}_{x \geq 0}\right)}{2(b+\gamma)}-\beta r\right) V_{r}+\frac{\left(a+\gamma r+\alpha x \mathbb{1}_{x \geq 0}\right)^{2}}{4(b+\gamma)}-H(x)+\frac{1}{2} \sigma^{2} V_{x x} .
\end{aligned}
$$

To solve (4.5), using the method of undetermined coefficients, we conjecture a polynomial form of the profitto-go function in the following proposition.

Proposition 4.1. The following expression satisfies the HJB equation associated with problem (3.5):

$$
V(x, r)=a_{1}+a_{2} x+a_{3} x^{2}+a_{4} x r+a_{5} r+a_{6} r^{2},
$$

where coefficients $a_{i}, i=1, \ldots, 6$ are given in Appendix $A$.

Proposition 4.1 presents the firm's value function being quadratic in the inventory and reference price, which is not surprising as it is well-known that such a standard stochastic linear-quadratic optimal control problem (i.e., the diffusion equation is linear in both state and control variables and the objective function is quadratic) admits quadratic value function (see, e.g., [32]). With respect to Proposition 4.1, for $x \geq 0$ (or $x<0$ ), we derive two Riccati systems consists of six non-linear algebraic equations (see Appendix A) that determine $a_{i}$ (or $\tilde{a}_{i}$ ), $i=1, \ldots, 6$, respectively. As a result, equations (4.3) and (4.4) become

$$
\begin{aligned}
& u^{*}=\frac{1}{2 c}\left(a_{2}+2 a_{3} x+a_{4} r\right), \\
& p^{*}=\frac{a+\gamma r+\alpha x \mathbb{1}_{x \geq 0}}{2(b+\gamma)}+\frac{a_{2}+2 a_{3} x+a_{4} r}{2}+\frac{\beta\left(a_{4} x+a_{5}+2 a_{6} r\right)}{2(b+\gamma)},
\end{aligned}
$$

then we have the following corollary.

Corollary 4.2. With the real-valued constants $a_{1}-a_{6}$ and $\tilde{a}_{1}-\tilde{a}_{6}$ satisfying the Riccati systems in Appendix B, 
(i) when $x(t) \geq 0$, if $a_{2}+2 a_{3} x+a_{4} r \geq 0$ and $2(b+\gamma) p \leq \alpha_{1}+\left(\alpha+\alpha_{2}\right) x+\alpha_{3} r \leq 2(b+\gamma) \bar{p}$, the optimal feedback replenishment rate and selling price are

$$
\begin{aligned}
& u^{*}(x, r)=\frac{1}{2 c}\left(a_{2}+2 a_{3} x+a_{4} r\right), \\
& p^{*}(x, r)=\frac{1}{2(b+\gamma)}\left(\alpha_{1}+\left(\alpha+\alpha_{2}\right) x+\alpha_{3} r\right),
\end{aligned}
$$

where $\alpha_{1}=a+(b+\gamma) a_{2}+\beta a_{5}, \alpha_{2}=2(b+\gamma) a_{3}+\beta a_{4}$ and $\alpha_{3}=\gamma+(b+\gamma) a_{4}+2 \beta a_{6}$. Otherwise, if $a_{2}+2 a_{3} x+a_{4} r<0$, then $u^{*}(x, r)=0$; if $\alpha_{1}+\left(\alpha+\alpha_{2}\right) x+\alpha_{3} r<2(b+\gamma) \underline{p}$, then $p^{*}(x, r)=p$; and if $\alpha_{1}+\left(\alpha+\alpha_{2}\right) x+\alpha_{3} r>2(b+\gamma) \bar{p}$, then $p^{*}(x, r)=\bar{p}$;

(ii) when $x(t)<0$, if $\tilde{a}_{2}+2 \tilde{a}_{3} x+\tilde{a}_{4} r \geq 0$ and $2(b+\gamma) \underline{p} \leq \tilde{\alpha}_{1}+\tilde{\alpha}_{2} x+\tilde{\alpha}_{3} r \leq 2(b+\gamma) \bar{p}$, the optimal feedback replenishment rate and selling price are

$$
\begin{aligned}
& u^{*}(x, r)=\frac{1}{2 c}\left(\tilde{a}_{2}+2 \tilde{a}_{3} x+\tilde{a}_{4} r\right), \\
& p^{*}(x, r)=\frac{1}{2(b+\gamma)}\left(\tilde{\alpha}_{1}+\tilde{\alpha}_{2} x+\tilde{\alpha}_{3} r\right),
\end{aligned}
$$

where $\tilde{\alpha}_{1}=a+(b+\gamma) \tilde{a}_{2}+\beta \tilde{a}_{5}, \tilde{\alpha}_{2}=2(b+\gamma) \tilde{a}_{3}+\beta \tilde{a}_{4}$ and $\tilde{\alpha}_{3}=\gamma+(b+\gamma) \tilde{a}_{4}+2 \beta \tilde{a}_{6}$. Otherwise, if $\tilde{a}_{2}+2 \tilde{a}_{3} x+\tilde{a}_{4} r<0$, then $u^{*}(x, r)=0$; if $\tilde{\alpha}_{1}+\tilde{\alpha}_{2} x+\tilde{\alpha}_{3} r<2(b+\gamma) \underline{p}$, then $p^{*}(x, r)=\underline{p}$; and if $\tilde{\alpha}_{1}+\tilde{\alpha}_{2} x+\tilde{\alpha}_{3} r>2(b+\gamma) \bar{p}$, then $p^{*}(x, r)=\bar{p}$.

Using verification theorem (see, e.g., [23], Thm. 4.1 in Chap. VI), one can easily verify that $u^{*}(x, r)$ and $p^{*}(x, r)$ (in Cor. 4.2) solved in this way is indeed optimal and $V(x, r)$ is given by (4.6). As is customary in an infinite-horizon control problem, the firm can choose stationary feedback strategies, that is, the replenishment and pricing strategies depend on the states but not on time (explicitly). Corollary 4.2 suggests that the optimal replenishment rate and pricing strategies both are linearly dependent on the two observed state variables $(x, r)$ when the current inventory level $x$ is positive or negative, which provides quantitative relationships between optimal policies and system parameters. The linear feedback solutions present a closed-loop strategy-making approach which allows the manager to dynamically adjust his replenishment and pricing strategies in response to any change of the inventory and reference price levels, and thus it serves as a powerful tool to support managers in making operational decisions. Note that although the replenishment and price decisions both are deterministic functions of $x$ and $r$, the intertemporal replenishment path $u^{*}(x(t), r(t))$ and price path $p^{*}(x(t), r(t))$ will be random because the inventory level $x(t)$ stochastically evolves over time. Thus, we can not specify deterministically the temporal replenishment and price paths aforehand. In the following numerical section, we will simulate the sample paths of optimal solutions to further illustrate Corollary 4.2.

In the following analysis, we assume that the systems admit interior solutions. Substituting the optimal strategies (4.7), (4.8) and (4.9), (4.10) into the state equations (3.1) and (3.4), respectively, yields two systems of two linear differential equations.

If $x \geq 0$,

$$
\begin{aligned}
\mathrm{d} x & =\left[\frac{1}{2 c}\left(a_{2}+2 a_{3} x+a_{4} r\right)-a+\frac{1}{2}\left(\alpha_{1}+\left(\alpha+\alpha_{2}\right) x+\alpha_{3} r\right)-\gamma r-(\theta+\alpha) x\right] \mathrm{d} t+\sigma \mathrm{d} W, \\
\mathrm{~d} r & =\beta\left[\frac{1}{2(b+\gamma)}\left(\alpha_{1}+\left(\alpha+\alpha_{2}\right) x+\alpha_{3} r\right)-r\right] \mathrm{d} t,
\end{aligned}
$$

and if $x<0$,

$$
\begin{aligned}
\mathrm{d} x & =\left[\frac{1}{2 c}\left(\tilde{a}_{2}+2 \tilde{a}_{3} x+\tilde{a}_{4} r\right)-a+\frac{1}{2}\left(\tilde{\alpha}_{1}+\tilde{\alpha}_{2} x+\tilde{\alpha}_{3} r\right)-\gamma r\right] \mathrm{d} t+\sigma \mathrm{d} W, \\
\mathrm{~d} r & =\beta\left[\frac{1}{2(b+\gamma)}\left(\tilde{\alpha}_{1}+\tilde{\alpha}_{2} x+\tilde{\alpha}_{3} r\right)-r\right] \mathrm{d} t .
\end{aligned}
$$


Applying the expectation operation on both sides of the above systems and making a simplification, we have for case $x \geq 0$,

$$
\begin{aligned}
& \dot{X}=\frac{a_{2}}{2 c}-a+\frac{1}{2} \alpha_{1}+\left(\frac{a_{3}}{c}+\frac{1}{2}\left(\alpha+\alpha_{2}\right)-(\theta+\alpha)\right) X+\left(\frac{a_{4}}{2 c}+\frac{1}{2} \alpha_{3}-\gamma\right) R, \\
& \dot{R}=\frac{\beta \alpha_{1}}{2(b+\gamma)}+\frac{\beta\left(\alpha+\alpha_{2}\right)}{2(b+\gamma)} X+\left(\frac{\beta \alpha_{3}}{2(b+\gamma)}-\beta\right) R,
\end{aligned}
$$

and for case $x<0$,

$$
\begin{aligned}
& \dot{X}=\frac{\tilde{a}_{2}}{2 c}-a+\frac{1}{2} \tilde{\alpha}_{1}+\left(\frac{\tilde{a}_{3}}{c}+\frac{1}{2} \tilde{\alpha}_{2}\right) X+\left(\frac{\tilde{a}_{4}}{2 c}+\frac{1}{2} \tilde{\alpha}_{3}-\gamma\right) R, \\
& \dot{R}=\frac{\beta \tilde{\alpha}_{1}}{2(b+\gamma)}+\frac{\beta \tilde{\alpha}_{2}}{2(b+\gamma)} X+\left(\frac{\beta \tilde{\alpha}_{3}}{2(b+\gamma)}-\beta\right) R,
\end{aligned}
$$

where $X=E(x)$ and $R=E(r)$.

The next proposition characterizes the steady-state equilibria.

Proposition 4.3. Under the optimal policy (4.7) to (4.10),

(i) the optimal expected steady-state equilibria are given by:

if $x \geq 0$,

$$
\begin{aligned}
u^{s s} & =\frac{1}{2 c}\left(a_{2}+2 a_{3} X^{s s}+a_{4} R^{s s}\right) \\
p^{s s} & =R^{s s}=\frac{-\alpha_{1}\left(2 a_{3}+c\left(\alpha+\alpha_{2}\right)-2 c(\alpha+\theta)\right)-\left(\alpha+\alpha_{2}\right)\left(2 c a-a_{2}-c \alpha_{1}\right)}{\left(2 a_{3}+c\left(\alpha+\alpha_{2}\right)-2 c(\alpha+\theta)\right)\left(\alpha_{3}-2(b+\gamma)\right)-\left(\alpha+\alpha_{2}\right)\left(a_{4}+c \alpha_{3}-2 c \gamma\right)}, \\
X^{s s} & =\frac{\left(2 c a-a_{2}-c \alpha_{1}\right)\left(\alpha_{3}-2(b+\gamma)\right)+\alpha_{1}\left(a_{4}+c \alpha_{3}-2 c \gamma\right)}{\left(2 a_{3}+c\left(\alpha+\alpha_{2}\right)-2 c(\alpha+\theta)\right)\left(\alpha_{3}-2(b+\gamma)\right)-\left(\alpha+\alpha_{2}\right)\left(a_{4}+c \alpha_{3}-2 c \gamma\right)}, \\
V^{s s} & =a_{1}+a_{2} X^{s s}+a_{3}\left(X^{s s}\right)^{2}+a_{4} X^{s s} R^{s s}+a_{5} R^{s s}+a_{6}\left(R^{s s}\right)^{2},
\end{aligned}
$$

and if $x<0$,

$$
\begin{aligned}
u^{s s} & =\frac{1}{2 c}\left(\tilde{a}_{2}+2 \tilde{a}_{3} X^{s s}+\tilde{a}_{4} R^{s s}\right), \\
p^{s s} & =R^{s s}=\frac{-\tilde{\alpha}_{1}\left(2 \tilde{a}_{3}+c \tilde{\alpha}_{2}\right)-\tilde{\alpha}_{2}\left(2 c a-\tilde{a}_{2}-c \tilde{\alpha}_{1}\right)}{\left(2 \tilde{a}_{3}+c \tilde{\alpha}_{2}\right)\left(\tilde{\alpha}_{3}-2(b+\gamma)\right)-\tilde{\alpha}_{2}\left(\tilde{a}_{4}+c \tilde{\alpha}_{3}-2 c \gamma\right)}, \\
X^{s s} & =\frac{\left(2 c a-\tilde{a}_{2}-c \tilde{\alpha}_{1}\right)\left(\tilde{\alpha}_{3}-2(b+\gamma)\right)+\tilde{\alpha}_{1}\left(\tilde{a}_{4}+c \tilde{\alpha}_{3}-2 c \gamma\right)}{\left(2 \tilde{a}_{3}+c \tilde{\alpha}_{2}\right)\left(\tilde{\alpha}_{3}-2(b+\gamma)\right)-\tilde{\alpha}_{2}\left(\tilde{a}_{4}+c \tilde{\alpha}_{3}-2 c \gamma\right)}, \\
V^{s s} & =\tilde{a}_{1}+\tilde{a}_{2} X^{s s}+\tilde{a}_{3}\left(X^{s s}\right)^{2}+\tilde{a}_{4} X^{s s} R^{s s}+\tilde{a}_{5} R^{s s}+\tilde{a}_{6}\left(R^{s s}\right)^{2},
\end{aligned}
$$

where the superscript "ss" stands for steady-state;

(ii) the steady-states in (i) is independent of the initial inventory level $x_{0}$ and reference price $r_{0}$.

Proof. See Appendix B.

When the planning horizon is infinite, it makes sense to focus on the long-run behaviors of the system. Thus, Proposition 4.3 provides the expected steady-state results of the optimal strategies, state variables, as well as profit, which allows us to perform comparative statics analysis to examine the sensitivity of the equilibria 
TABLE 3. Baseline parameters.

\begin{tabular}{llllllllllllll}
\hline \hline Parameter & $a$ & $b$ & $\gamma$ & $\beta$ & $\theta$ & $\alpha$ & $h$ & $s$ & $c$ & $\sigma$ & $\rho$ & $x_{0}$ & $r_{0}$ \\
\hline Value & 1 & 0.1 & 2 & 0.5 & 0.03 & 0.1 & 2 & 3 & 2 & 0.6 & 0.01 & 5 & 3 \\
\hline
\end{tabular}

to major model parameters in the next section as a standard analytical method is not available to us. At the expected equilibria, the reference price effect would disappear (i.e., $p^{s s}=R^{s s}$ ). However, note that the parameter $\gamma$ does play a role in driving the dynamics to their steady-states. For notation convenience, for $x \geq 0$ (resp. $x<0$ ) we denote by $J$ (resp. $\widetilde{J}$ ) the Jacobian matrix for $(4.11)$ and $(4.12)$ (resp. (4.13) and (4.14)), by $\operatorname{det}(J)(\operatorname{resp} . \operatorname{det}(J))$ its determinant, and by $\operatorname{tr}(J)($ resp. $\operatorname{tr}(\tilde{J}))$ its trace. Then we conclude the stability and convergence properties of the expected equilibria in Proposition 4.4.

Proposition 4.4. For the problem (3.5), when $x \geq 0$, if

$$
\begin{array}{r}
\left(2 a_{3}+c\left(\alpha+\alpha_{2}\right)-2 c(\alpha+\theta)\right)\left(\alpha_{3}-2(b+\gamma)\right)-\left(\alpha+\alpha_{2}\right)\left(a_{4}+c \alpha_{3}-2 c \gamma\right)>0 \\
\left(2 a_{3}+c\left(\alpha+\alpha_{2}\right)-2 c(\alpha+\theta)\right)(b+\gamma)+\beta c\left(\alpha_{3}-2(b+\gamma)\right)<0
\end{array}
$$

and when $x<0$, if

$$
\begin{aligned}
\left(2 \tilde{a}_{3}+c \tilde{\alpha}_{2}\right)\left(\tilde{\alpha}_{3}-2(b+\gamma)\right)-\tilde{\alpha}_{2}\left(\tilde{a}_{4}+c \tilde{\alpha}_{3}-2 c \gamma\right) & >0, \\
\left(2 \tilde{a}_{3}+c \tilde{\alpha}_{2}\right)(b+\gamma)+\beta c\left(\tilde{\alpha}_{3}-2(b+\gamma)\right) & <0,
\end{aligned}
$$

the expected equilibria $X^{s s}$ and $R^{s s}$ are globally asymptotically stable. Furthermore, if $(\operatorname{tr}(J))^{2}-4 \operatorname{det}(J) \geq 0$ $\left(\right.$ resp. $\left.(\operatorname{tr}(\tilde{J}))^{2}-4 \operatorname{det}(\tilde{J}) \geq 0\right), X^{s s}$ and $R^{s s}$ are monotonically convergent, otherwise they converge with transient oscillations.

Proof. See Appendix C.

Stability means that the market state is stationary, and the firm can obtain fixed profit under fixed replenishment rate and price at any time. From the perspective of decision-making, stability is beneficial for firms to make long-term strategies. Proposition 4.4 presents sufficient conditions for the stability of the expected long-run behavior, which guarantees globally asymptotically stable expected equilibria those are preferred in infinite horizon control problems. Thus, all the expected variables converge to the steady-states outcomes in Proposition 4.3 as time goes to infinity regardless of the initial conditions.

\section{NUMERICAL ANALYSIS}

Since the analytical solutions to the two Ricatti systems in Appendix A that determine the coefficients of the retailer's value functions are unavailable to us, we resort to numerical experiments to illustrate the theoretical results and perform sensitivity analysis to gain some managerial insights into the optimal strategies and profit.

\subsection{A baseline example}

The following illustrative example provides certain insights into the problem studied.

Our model has 13 parameters: the market potential $a$; the price sensitivity parameter $b$ and the reference effect intensity $\gamma$; the memory factor $\beta$; the deterioration parameter $\theta$; the parameter $\alpha$; the holding and shortage cost coefficients $h$ and $s$; the replenishment cost coefficient $c$; the demand uncertainty $\sigma$; the discount rate $\rho$; and the initial inventory level $x_{0}$ and reference price $r_{0}$. These baseline parameter values are reported in Table 3 .

Given these parameter values, if $x \geq 0(x<0)$, we have $a_{1}=122.4609048\left(\tilde{a}_{1}=113.2822215\right)$, $a_{2}=-0.6424352729\left(\tilde{a}_{2}=-0.7255920623\right), a_{3}=-0.8895157324\left(\tilde{a}_{3}=-1.119395878\right), a_{4}=0.5015916239$ 
$\left(\tilde{a}_{4}=0.4937901151\right), a_{5}=6.044926586\left(\tilde{a}_{5}=6.04867787\right), a_{6}=0.6860917648\left(\tilde{a}_{6}=0.6867207651\right)$ satisfy the Ricatti systems. As a result, $\alpha_{1}=2.673349220\left(\tilde{\alpha}_{1}=2.500595607\right), \alpha_{2}=-3.485170264\left(\tilde{\alpha}_{2}=-4.454567630\right)$, $\alpha_{3}=3.739434175\left(\tilde{\alpha}_{3}=3.723680007\right)$. By Corollary 4.2, the optimal interior replenishment rate and selling price are given, respectively, by

$$
\begin{aligned}
& u^{*}(t)= \begin{cases}0.5-0.4447578662 x+0.1253979060 r, & \text { if } x \geqslant 0, \\
-0.1813980156-0.5596979390 x+0.1234475288 r, & \text { if } x<0,\end{cases} \\
& p^{*}(t)= \begin{cases}0.6365117191-0.8059929200 x+0.8903414703 r, & \text { if } x \geqslant 0, \\
0.5953799064-1.060611340 x+0.8865904779 r, & \text { if } x<0 .\end{cases}
\end{aligned}
$$

These optimal strategies say that the replenishment rate and selling price each negatively depends on the current inventory level $x$ and positively depends on the current reference price level $r$. This is intuitive. As the current inventory is higher, the firm should have a lower replenishment rate to save operation costs and set a lower sales price to stimulate demand. As the current reference price grows, which results in a high demand, the firm should make more orders and can set a higher price to make more profit.

Furthermore, if $x \geq 0(x<0)$, we calculate $\operatorname{det}(J)=0.1223481095(\operatorname{det}(\tilde{J})=0.1502330295)$, $\operatorname{tr}(J)=-2.322172264(\operatorname{tr}(\tilde{J})=-2.843686514)$, and $(\operatorname{tr}(J))^{2}-4 \operatorname{det}(J)=4.903091586>0\left((\operatorname{tr}(\tilde{J}))^{2}-\right.$ $4 \operatorname{det}(\tilde{J})=7.485620872>0)$. By Propositions 4.3 and 4.4 , the expected steady states, $\left(X^{s s}, R^{s s}\right)=$ $(0.066195350,5.317951430)$ if $x \geq 0$ or $\left(X^{s s}, R^{s s}\right)=(-0.003147163,5.279255319)$ if $x<0$, are globally asymptotically stable and the convergence to these states is monotonic.

By virtue of the (stochastic) Euler discretion schemes (see [13]), the solid lines in Figures 1(a)-1(d) depict the sample paths of the optimal strategies and states over 150 time units for a low initial reference price $r_{0}=3$ and a high initial reference price $r_{0}=8$, respectively. Several observations are summarized as follows. Firstly, we observe that the firm always replenishes/produces (i.e., $\left.u^{*}>0\right)$ and the selling price and the reference price are kept within a positive range of values (i.e., $p^{*}>0$ and $r^{*}>0$ ).

Secondly, the consumers' initial reference price has an important influence on the retailer's sales and replenishment plan. The retailer may adopt different replenishment and pricing strategies based on different initial reference price. In particular, under the optimal strategies (5.1) and (5.2), the sample paths of optimal inventory level $x^{*}(t)$ shown in Figure 1(a) have similar evolution trends when $r_{0}=3$ and $r_{0}=8$. Both inventory paths start from an initial starting point of $x_{0}=5$ and fall sharply over time at the initial sales periods due to demand and deterioration, and are then stable at about 0.0662. From Figures $1(\mathrm{~b})$ and $1(\mathrm{c})$, we find that $u^{*}(t)$ and $p^{*}(t)$ have similar evolution trends when $r_{0}=3$ or $r_{0}=8$. When $r_{0}=3, u^{*}(t)$ increases gradually in the beginning and then hovers around $u^{*}(t)=0.4768$, and the firm adopts a price-penetration strategy. In other words, when the initial reference price is low and the inventory level is high in the initial stage, the retailer should keep a lower price to increase the demand to reduce inventory, which ultimately results in lower inventory cost and the optimal profit. In contrast, when $r_{0}=8, u^{*}(t)$ firstly rises and then drops until it stabilizes at about 0.4768 . In the meantime, the firm may take a price-penetration strategy in the beginning and then take a price-skimming strategy. That is, when the initial reference price is high, the retailer would set a higher product price to establish a higher reference price in order to benefit from the increased demand which offsets the higher replenishment cost and thus the retailer obtains the optimal profit. As a consequence, it is essential for the firm to examine the initial reference price $r_{0}$ so as to make the best joint replenishment and pricing decisions. Furthermore, when $r_{0}=3$, the sample path of reference price in Figure 1(d) firstly decreases and then gradually increases until it stabilizes at about 5.3180, while when $r_{0}=8, r^{*}(t)$ decreases and stabilizes in the long run. In addition, comparing Figure 1(d) with Figure 1(c), we find that reference price dynamics do not systematically imitate sales price dynamics. This result is different from previous findings that the selling price and reference price changes in the same way throughout the whole time horizon, as shown in $[22,57]$. This is because we consider the replenishment/production-inventory decision in the evolution of the reference price and decisions.

Finally, when $\sigma^{2}=0$, the smooth dashed lines in Figures 1(a)-1(d) characterize the optimal deterministic strategies $\left(i . e ., u_{d}^{*}(t)\right.$ and $\left.p_{d}^{*}(t)\right)$ and state trajectories $\left(i . e ., x_{d}^{*}(t)\right.$ and $\left.r_{d}^{*}(t)\right)$ when $r_{0}=3$ and $r_{0}=8$, respectively. 


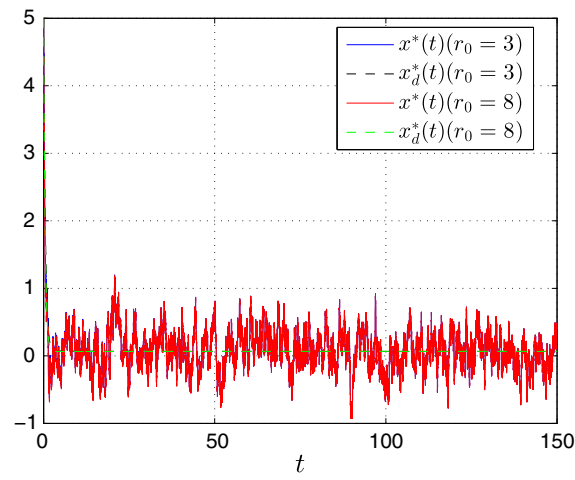

(a)

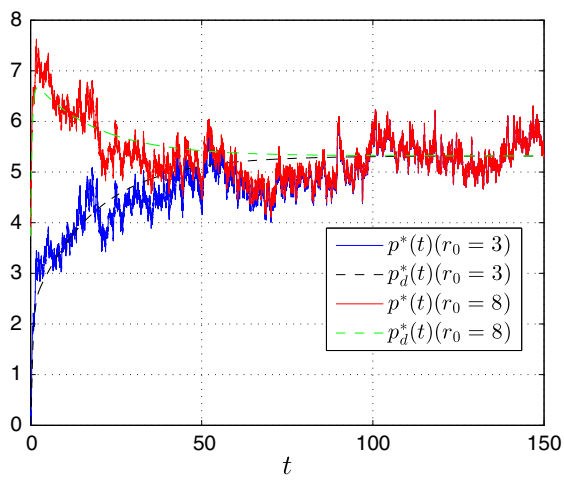

(c)

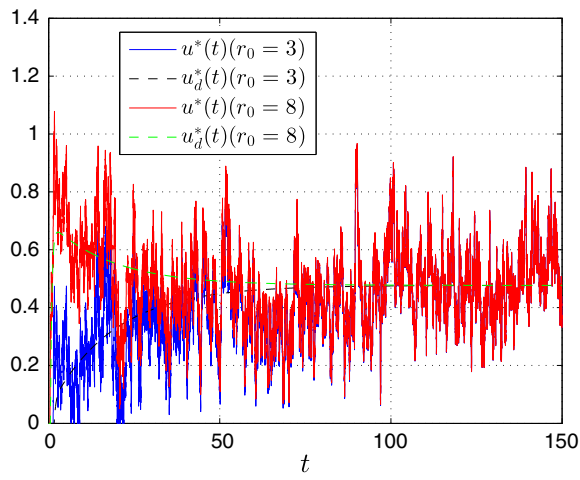

(b)

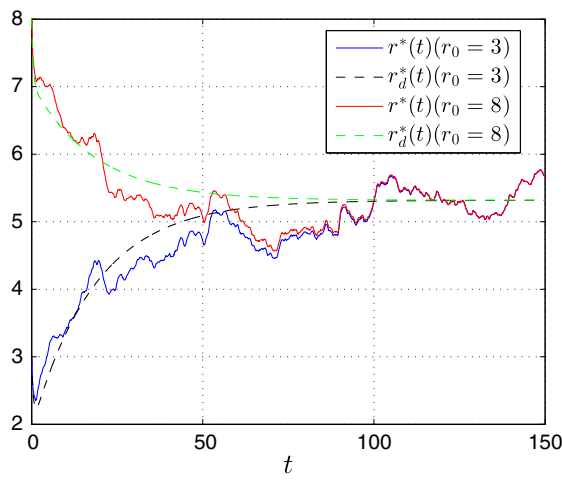

(d)

Figure 1. The optimal (a) inventory level $x^{*}(t)$, (b) replenishment rate $u^{*}(t)$, (c) selling price $p^{*}(t)$, and (d) reference price $r^{*}(t)$.

As the demand uncertainty tends to vanish, the inventory and reference price levels are more smooth, which results that the optimal selling price and replenishment/production rate are more smooth since the optimal strategies are in the feedback form of the state variables. It is shown that the deterministic system has similar evolution trends as the stochastic system.

\subsection{Sensitivity analysis}

It makes sense to focus on the long-run behavior of the optimal strategies and profit since this paper assumes an infinite planning horizon. In this subsection, we conduct a sensitivity analysis of the key model parameters and show these results in Table 4. In each experiment, we obtain the steady-state values varying one parameter value while remaining other parameter values at their baseline levels.

Table 5 reports the variations in steady-state values. For each parameter, we consider three values, e.g., 0.08, 0.1 and 0.12 for $\alpha$. An up-arrow means that a positive relationship between the expected steady-state value taken by the corresponding variable when the parameter varies from the lowest value to the highest one. In contrast, a down-arrow sign denotes a negative relationship.

The results in Tables 4 and 5 allow for the following comments:

(1) Impact of demand uncertainty parameter $\sigma$. The increase of the additive form of demand uncertainty does not affect the optimal expected steady states, i.e., $u^{s s}, p^{s s}, X^{s s}, R^{s s}$ and $D^{s s}$, which is typical results of stochastic linear-quadratic control models under uncertainty involved in the additive demand (see similar 
TABLE 4. Sensitivity analysis with respect to model parameters.

\begin{tabular}{lllllll}
\hline \hline & PV & $u^{s s}$ & $p^{s s}\left(=R^{s s}\right)$ & $X^{s s}$ & $D^{s s}$ & $V^{s s}$ \\
\hline \multirow{4}{*}{$\sigma$} & 0.5 & 0.4768 & 5.3180 & 0.0662 & 0.4748 & 183.9254 \\
& 0.6 & 0.4768 & 5.3180 & 0.0662 & 0.4748 & 174.1408 \\
& 0.7 & 0.4768 & 5.3180 & 0.0662 & 0.4748 & 162.5771 \\
\hline \multirow{4}{*}{$\gamma$} & 1.5 & 0.4652 & 5.4396 & 0.0709 & 0.4631 & 172.0973 \\
& 2 & 0.4768 & 5.3180 & 0.0662 & 0.4748 & 174.1408 \\
& 2.5 & 0.4873 & 5.2072 & 0.0620 & 0.4855 & 175.1596 \\
\hline \multirow{3}{*}{$\beta$} & 0.4 & 0.4871 & 5.2097 & 0.0621 & 0.4852 & 172.8504 \\
& 0.5 & 0.4768 & 5.3180 & 0.0662 & 0.4748 & 174.1408 \\
& 0.6 & 0.4693 & 5.3964 & 0.0692 & 0.4673 & 174.8261 \\
\hline \multirow{4}{*}{$\alpha$} & 0.08 & 0.4751 & 5.3027 & 0.0490 & 0.4736 & 173.3693 \\
& 0.1 & 0.4768 & 5.3180 & 0.0662 & 0.4748 & 174.1408 \\
& 0.12 & 0.4788 & 5.3369 & 0.0835 & 0.4763 & 175.0823 \\
\hline \multirow{2}{*}{$\theta$} & 0.02 & 0.4766 & 5.3194 & 0.0710 & 0.4752 & 174.1524 \\
& 0.03 & 0.4768 & 5.3180 & 0.0662 & 0.4748 & 174.1408 \\
& 0.04 & 0.4769 & 5.3163 & 0.0614 & 0.4745 & 174.1352 \\
\hline
\end{tabular}

Notes. PV denotes parameter value.

TABLE 5. Variations in steady-state values.

\begin{tabular}{llllll}
\hline \hline & $u^{s s}$ & $p^{s s}\left(R^{s s}\right)$ & $X^{s s}$ & $D^{s s}$ & $V^{s s}$ \\
\hline$\sigma(0.5 ; 0.6 ; 0.7)$ & - & - & - & - & $\downarrow$ \\
$\gamma(1.5 ; 2 ; 2.5)$ & $\uparrow$ & $\downarrow$ & $\downarrow$ & $\uparrow$ & $\uparrow$ \\
$\beta(0.4 ; 0.5 ; 0.6)$ & $\downarrow$ & $\uparrow$ & $\uparrow$ & $\downarrow$ & $\uparrow$ \\
$\alpha(0.08 ; 0.1 ; 0.12)$ & $\uparrow$ & $\uparrow$ & $\uparrow$ & $\uparrow$ & $\uparrow$ \\
$\theta(0.02 ; 0.03 ; 0.04)$ & $\uparrow$ & $\downarrow$ & $\downarrow$ & $\downarrow$ & $\downarrow$ \\
\hline
\end{tabular}

Notes. - means the value unchanged.

results in $[31,32])$. However, the demand uncertainty has a negative effect on the optimal expected steady profit $V^{s s}$. A higher demand noise intensity always leads to a lower total expected profit under our problem setting, which is intuitive and expected.

(2) Impact of reference effect intensity $\gamma$. The demand function is independent of the reference effect since the term $p-r$ vanishes at the expected steady-state. As $\gamma$ increases, at expected steady-state, both price and inventory level descend, whereas replenishment rate and demand, as well as profit ascend. In other words, when consumers have a lower sensitivity to their reference price (i.e., a lower $\gamma$ ), the firm should set a higher price which induces a lower replenishment rate, as a result of a lower demand since the negative price effect has a greater negative impact on demand than the positive effect of display stock level on demand. The low sales decrease the firm's optimal profit.

(3) Impact of memory factor $\beta$. The memory parameter $\beta$ has an opposite impact on all expected steadystates compared with reference effect intensity $\gamma$ except the steady-state profit. The messages drawn out of the above tables are as follows: as $\beta$ increases, meaning that consumers have a poorer (i.e., more shortterm) memory or are less loyalty to the product, the firm has an opportunity to raise his sales price to increase revenue which offsets the higher holding cost (resulted from higher inventory level) and the lower replenishment cost (resulted from lower replenishment rate) as well as the deterioration loss.

(4) Impact of parameter $\alpha$. Increasing parameter $\alpha$ leads to increased demand and thus higher replenishment rate. This is intuitive because the displayed stock level stimulates sales. The firm can set a relatively higher 
price maybe because of the positive effect on demand due to the higher displayed inventory level dominates the negative effect on demand owing to a higher price. Finally, the firm obtains a higher profit since the increased revenue offsets the higher replenishment and holding costs.

(5) Impact of constant deterioration rate $\theta$. The results show that the expected steady-state (reference) price, inventory level, demand, and profit decrease as the constant deterioration rate $\theta$ increases, whereas $\theta$ has a positive effect on the expected steady-state replenishment rate. The chain of effects may be explained as follows: On the one hand, excess inventory waste due to higher deterioration rate induces the firm to set a higher replenishment rate in order to remain stock level. On the other hand, the deteriorating nature of products forces the firm to lower his price to boost demand and expend the inventory. However, the demand reduction due to lower inventory level (which has a positive effect on demand) outweighs the increases in demand owing to lower price. Ultimately, the lower demand and price translate into lower firm profitability.

\section{Conclusions}

This is the first paper in the literature to investigate joint stochastic dynamic replenishment/ordering and pricing policies for deteriorating items in the presence of reference price and stock display effects simultaneously in a continuous-time framework. The market potential is subject to some exogenous noise and follows a stochastic process. The demand rate negatively depends on selling price, positively on reference price and displayed stock quantity. Shortages are fully backlogged in this model. First of all, we formulate this problem as a stochastic optimal control model with the objective that maximizes the total profit. Based on the principle of optimality, it turns out that the optimization problem is converted to solve a HJB equation. Then, we show that a two-satesdependent feedback policy is optimal. We also show the existence of the expected steady states and provide sufficient conditions for the asymptotic behavior of the system. Finally, an illustrative example is provided to demonstrate the theoretical results. We also carry out a sensitivity analysis to assess the impact of the key model parameters on the optimal expected equilibrium and propose some useful managerial insights. There are several avenues in terms of extensions. First, one could consider the finite replenishment capacity and shelf space without shortage in our model. Second, a potential research direction is to model multi-products competition. Third, one could introduce some operating and promotion activities, e.g., preservation technology investment and advertising, as decision variables into our model. Finally, it will be worthwhile considering asymmetric reference price effect in our model which results in a non-smooth optimization problem.

\section{Appendix A. The Riccati System}

From (4.6), we have $V_{x}=a_{2}+2 a_{3} x+a_{4} r, V_{x x}=2 a_{3}$ and $V_{r}=a_{4} x+a_{5}+2 a_{6} r$. Inserting them into (4.5) yields

$$
\begin{aligned}
\rho V= & \left(\left(\frac{1}{4 c}+\frac{b+\gamma}{4}\right) 4 a_{3}^{2}+\frac{\beta^{2}}{4(b+\gamma)} a_{4}^{2}+\beta a_{3} a_{4}\right) x^{2}+\left(\left(\frac{1}{4 c}+\frac{b+\gamma}{4}\right) a_{4}^{2}+\frac{\beta^{2}}{4(b+\gamma)} 4 a_{6}^{2}+\beta a_{4} a_{6}\right) r^{2} \\
& +\left(\left(\frac{1}{4 c}+\frac{b+\gamma}{4}\right) 4 a_{3} a_{4}+\frac{\beta^{2}}{4(b+\gamma)} 4 a_{4} a_{6}+\frac{\beta}{2}\left(4 a_{3} a_{6}+a_{4}^{2}\right)\right) x r+\left(\left(\frac{1}{4 c}+\frac{b+\gamma}{4}\right) 4 a_{2} a_{3}\right. \\
& \left.+\frac{\beta^{2}}{4(b+\gamma)} 2 a_{4} a_{5}+\frac{\beta}{2}\left(2 a_{3} a_{5}+a_{2} a_{4}\right)\right) x+\left(\left(\frac{1}{4 c}+\frac{b+\gamma}{4}\right) 2 a_{2} a_{4}+\frac{\beta^{2}}{4(b+\gamma)} 2 a_{5} a_{6}+\frac{\beta}{2}\left(2 a_{2} a_{6}+a_{4} a_{5}\right)\right) r \\
& +\left(\frac{1}{4 c}+\frac{b+\gamma}{4}\right) a_{2}^{2}+\frac{\beta^{2}}{4(b+\gamma)} a_{5}^{2}+\frac{\beta}{2} a_{2} a_{5}-\frac{a+\gamma r+(\alpha+2 \theta) x \mathbb{1}_{x \geq 0}}{2}\left(a_{2}+2 a_{3} x+a_{4} r\right)-H(x)+a_{3} \sigma^{2} \\
& +\left(\frac{\beta\left(a+\gamma r+\alpha x \mathbb{1}_{x \geq 0}\right)}{2(b+\gamma)}-\beta r\right)\left(a_{4} x+a_{5}+2 a_{6} r\right)+\frac{\left(a+\gamma r+\alpha x \mathbb{1}_{x \geq 0}\right)^{2}}{4(b+\gamma)} .
\end{aligned}
$$


The above equation should hold for any $x$ and $r$, when $x \geq 0$, using $V(x, r)$ in $(4.6)$ and $H(x)=h x^{2}$, we have the following Racatti system

$$
\begin{aligned}
4(b+\gamma) c \rho a_{1}= & {\left[b+\gamma+c(b+\gamma)^{2}\right] a_{2}^{2}+c \beta^{2} a_{5}{ }^{2}+2 c(b+\gamma) \beta a_{2} a_{5}-2 c(b+\gamma) a_{2} a } \\
& +2 c a_{5} \beta a+c a^{2}+4 c(b+\gamma) a_{3} \sigma^{2}, \\
4(b+\gamma) c \rho a_{2}= & {\left[b+\gamma+c(b+\gamma)^{2}\right] 4 a_{2} a_{3}+2 c \beta^{2} a_{4} a_{5}+2 c(b+\gamma) \beta\left(2 a_{3} a_{5}+a_{2} a_{4}\right) } \\
& -4 c(b+\gamma)\left(a_{3} a+\frac{a_{2}(\alpha+2 \theta)}{2}\right)+2 c\left(a_{4} \beta a+a_{5} \beta \alpha\right)+2 c \alpha a, \\
4(b+\gamma) c \rho a_{3}= & {\left[b+\gamma+c(b+\gamma)^{2}\right] 4 a_{3}^{2}+c \beta^{2} a_{4}^{2}+4 c(b+\gamma) \beta a_{3} a_{4}-4 c(b+\gamma) a_{3}(\alpha+2 \theta) } \\
& +2 c \beta a_{4} \alpha+c \alpha^{2}-4 c(b+\gamma) h, \\
4(b+\gamma) c \rho a_{4}= & {\left[b+\gamma+c(b+\gamma)^{2}\right] 4 a_{3} a_{4}+4 c \beta^{2} a_{4} a_{6}+2 c(b+\gamma) \beta\left(4 a_{3} a_{6}+a_{4}^{2}\right) } \\
& -4 c(b+\gamma)\left(a_{3} \gamma+\frac{a_{4}(\alpha+2 \theta)}{2}\right)+4 c a_{6} \beta \alpha+2 c a_{4} \beta \gamma-4 c(b+\gamma) a_{4} \beta+2 c \alpha \gamma, \\
4(b+\gamma) c \rho a_{5}= & {\left[b+\gamma+c(b+\gamma)^{2}\right] 2 a_{2} a_{4}+4 c \beta^{2} a_{5} a_{6}+2 c(b+\gamma) \beta\left(2 a_{2} a_{6}+a_{4} a_{5}\right) } \\
& -2 c(b+\gamma)\left(a_{2} \gamma+a_{4} a\right)+2 c\left[2 a_{6} \beta a+a_{5} \beta \gamma-2(b+\gamma) a_{5} \beta\right]+2 c a \gamma, \\
4(b+\gamma) c \rho a_{6}= & {\left[b+\gamma+c(b+\gamma)^{2}\right] a_{4}^{2}+4 c \beta^{2} a_{6}^{2}+4 c(b+\gamma) \beta a_{4} a_{6}-2 c(b+\gamma) a_{4} \gamma } \\
& +4 c a_{6} \beta \gamma-8 c(b+\gamma) a_{6} \beta+c \gamma^{2} .
\end{aligned}
$$

Similarly, when $x<0$, using $H(x)=s x^{2}$ and $\alpha=\theta=0$, we have

$$
\begin{aligned}
4(b+\gamma) c \rho \tilde{a}_{1}= & {\left[b+\gamma+c(b+\gamma)^{2}\right] \tilde{a}_{2}^{2}+c \beta^{2} \tilde{a}_{5}^{2}+2 c(b+\gamma) \beta \tilde{a}_{2} \tilde{a}_{5}-2 c(b+\gamma) \tilde{a}_{2} a } \\
& +2 c \tilde{a}_{5} \beta a+c a^{2}+4 c(b+\gamma) \tilde{a}_{3} \sigma^{2}, \\
4(b+\gamma) c \rho \tilde{a}_{2}= & {\left[b+\gamma+c(b+\gamma)^{2}\right] 4 \tilde{a}_{2} \tilde{a}_{3}+2 c \beta^{2} \tilde{a}_{4} \tilde{a}_{5}+2 c(b+\gamma) \beta\left(2 \tilde{a}_{3} \tilde{a}_{5}+\tilde{a}_{2} \tilde{a}_{4}\right) } \\
& -4 c(b+\gamma) \tilde{a}_{3} a+2 c \tilde{a}_{4} \beta a \\
4(b+\gamma) c \rho \tilde{a}_{3}= & {\left[b+\gamma+c(b+\gamma)^{2}\right] 4 \tilde{a}_{3}^{2}+c \beta^{2} \tilde{a}_{4}^{2}+4 c(b+\gamma) \beta \tilde{a}_{3} \tilde{a}_{4}-4 c(b+\gamma) s, } \\
4(b+\gamma) c \rho \tilde{a}_{4}= & {\left[b+\gamma+c(b+\gamma)^{2}\right] 4 \tilde{a}_{3} \tilde{a}_{4}+4 c \beta^{2} \tilde{a}_{4} \tilde{a}_{6}+2 c(b+\gamma) \beta\left(4 \tilde{a}_{3} \tilde{a}_{6}+\tilde{a}_{4}^{2}\right) } \\
& -4 c(b+\gamma) \tilde{a}_{3} \gamma+2 c \tilde{a}_{4} \beta \gamma-4 c(b+\gamma) \tilde{a}_{4} \beta \\
4(b+\gamma) c \rho \tilde{a}_{5}= & {\left[b+\gamma+c(b+\gamma)^{2}\right] 2 \tilde{a}_{2} \tilde{a}_{4}+4 c \beta^{2} \tilde{a}_{5} \tilde{a}_{6}+2 c(b+\gamma) \beta\left(2 \tilde{a}_{2} \tilde{a}_{6}+\tilde{a}_{4} \tilde{a}_{5}\right) } \\
& -2 c(b+\gamma)\left(\tilde{a}_{2} \gamma+\tilde{a}_{4} a\right)+2 c\left[2 \tilde{a}_{6} \beta a+\tilde{a}_{5} \beta \gamma-2(b+\gamma) \tilde{a}_{5} \beta\right]+2 c a \gamma, \\
4(b+\gamma) c \rho \tilde{a}_{6}= & {\left[b+\gamma+c(b+\gamma)^{2}\right] \tilde{a}_{4}^{2}+4 c \beta^{2} \tilde{a}_{6}^{2}+4 c(b+\gamma) \beta \tilde{a}_{4} \tilde{a}_{6}-2 c(b+\gamma) \tilde{a}_{4} \gamma } \\
& +4 c \tilde{a}_{6} \beta \gamma-8 c(b+\gamma) \tilde{a}_{6} \beta+c \gamma^{2} .
\end{aligned}
$$

where $\alpha_{1}-\alpha_{3}$ and $\tilde{\alpha}_{1}-\tilde{\alpha}_{3}$ are defined in Corollary 4.2. Unfortunately, these highly non-linear equations do not admit closed-form solutions for the unknowns $a_{1}-a_{6}$ and $\tilde{a}_{1}-\tilde{a}_{6}$, and therefore, we resort to the "solve" routine of Maple to solve them numerically.

\section{Appendix B. Proof of Proposition 4.3}

Setting $\dot{X}=\dot{R}=0$ in (4.11), (4.12) and (4.13), (4.14) and solving the corresponding two algebraic equations in the unknowns $X$ and $R$, respectively, we obtain the optimal expected steady states $X^{s s}$ and $R^{s s}$. Inserting them into (4.7) to (4.10) yields $u^{s s}$ and $p^{s s}$. Furthermore, taking expectation operation on both sides of (3.1) and setting the results to zero gives $p^{s s}=R^{s s}$. 


\section{Appendix C. Proof of Proposition 4.4}

If $x \geq 0$,

$$
\begin{aligned}
J & =\left(\begin{array}{ll}
\frac{a_{3}}{c}+\frac{1}{2}\left(\alpha+\alpha_{2}\right)-(\alpha+\theta) & \frac{a_{4}}{2 c}+\frac{1}{2} \alpha_{3}-\gamma \\
\frac{\beta\left(\alpha+\alpha_{2}\right)}{2(b+\gamma)} & \frac{\beta \alpha_{3}}{2(b+\gamma)}-\beta
\end{array}\right), \\
\operatorname{det}(J) & =\frac{\beta\left(2 a_{3}+c\left(\alpha+\alpha_{2}\right)-2 c(\alpha+\theta)\right)\left(\alpha_{3}-2(b+\gamma)\right)-\beta\left(\alpha+\alpha_{2}\right)\left(a_{4}+c \alpha_{3}-2 c \gamma\right)}{4 c(b+\gamma)}, \\
\operatorname{tr}(J) & =\frac{\left(2 a_{3}+c\left(\alpha+\alpha_{2}\right)-2 c(\alpha+\theta)\right)(b+\gamma)+\beta c\left(\alpha_{3}-2(b+\gamma)\right)}{2 c(b+\gamma)},
\end{aligned}
$$

and if $x<0$,

$$
\begin{aligned}
\tilde{J} & =\left(\begin{array}{ll}
\frac{\tilde{a}_{3}}{c}+\frac{\tilde{\alpha}_{2}}{2} & \frac{\tilde{a}_{4}}{2 c}+\frac{\tilde{\alpha}_{3}}{2}-\gamma \\
\frac{\beta \tilde{\alpha}_{2}}{2(b+\gamma)} & \frac{\beta \tilde{\alpha}_{3}}{2(b+\gamma)}-\beta
\end{array}\right), \\
\operatorname{det}(\tilde{J}) & =\frac{\beta\left(2 \tilde{a}_{3}+c \tilde{\alpha}_{2}\right)\left(\tilde{\alpha}_{3}-2(b+\gamma)\right)-\beta \tilde{\alpha}_{2}\left(\tilde{a}_{4}+c \tilde{\alpha}_{3}-2 c \gamma\right)}{4 c(b+\gamma)}, \\
\operatorname{tr}(\tilde{J}) & =\frac{\left(2 \tilde{a}_{3}+c \tilde{\alpha}_{2}\right)(b+\gamma)+\beta c\left(\tilde{\alpha}_{3}-2(b+\gamma)\right)}{2 c(b+\gamma)} .
\end{aligned}
$$

The proof is completed using the stability criteria in [51].

Acknowledgements. We would like to thank the two anonymous reviewers very much for their valuable comments and suggestions to improve this paper greatly. This work is supported by the National Natural Science Foundation of China [grant numbers 71771179; 71532015].

\section{REFERENCES}

[1] E. Adida and G. Perakis, A nonlinear continuous time optimal control model of dynamic pricing and inventory control with no backorders. Nav. Res. Logist. 54 (2007) 767-795.

[2] E. Adida and G. Perakis, Dynamic pricing and inventory control: uncertainty and competition. Oper. Res. 58 (2010) $289-302$.

[3] L. Arnold, Stochastic Differential Equations: Theory and Applications. John Wiley \& Sons, New York, NY (1974).

[4] H. Arslan and S. Kachani, Dynamic pricing under consumer reference-price effects, edited by J.J. Cochran. In: Wiley Encyclopedia of Operations Research and Management Science. John Wiley \& Sons, Hoboken, NJ (2011).

[5] R.C. Baker and T.L. Urban, A deterministic inventory system with an inventory level dependent demand rate. J. Oper. Res. Soc. 39 (1988) 823-831.

[6] R.A. Briesch, L. Krishnamurthi, T. Mazumdar and S.P. Raj, A comparative analysis of reference price models. J. Consum. Res. 24 (1997) 202-214.

[7] C.T. Chang, Inventory models with stock-dependent demand and nonlinear holding costs for deteriorating items. Asia Pac. J. Oper. Res. 21 (2004) 435-446.

[8] X. Chen and D. Simchi-Levi, Pricing and inventory management, edited by R. Philips and O. Ozalp. In: The Oxford Handbook of Pricing Management. Oxford University Press, Oxford (2012) 784-824.

[9] X. Chen, P. Hu and Z. Hu, Efficient algorithms for the dynamic pricing problem with reference price effect. Manage. Sci. 63 (2016) 4389-4408.

[10] X. Chen, P. Hu, S. Shum and Y. Zhang, Dynamic stochastic inventory management with reference price effects. Oper. Res. 64 (2016) 1529-1536.

[11] R. Chenavaz, Dynamic pricing with reference price dependence. Econ.: Open-Access Open-Assess. E-J. 10 (2016) 1-17.

[12] F.S. Chou and M. Parlar, Optimal control of a revenue management system with dynamic pricing facing linear demand. Optim. Control App. Methods. 27 (2006) 323-347.

[13] S. Cyganowski, P. Kloeden and J. Ombach, From Elementary Probability to Stochastic Differential Equations with MAPLE ${ }^{\circledR}$. Springer, New York, NY (2001).

[14] Y. Duan, Y. Cao and J. Huo, Optimal pricing, production, and inventory for deteriorating items under demand uncertainty: the finite horizon case. Appl. Math. Model. 58 (2018) 331-348. 
[15] C.Y. Dye and L.Y. Ouyang, An EOQ model for perishable items under stock-dependent selling rate and time-dependent partial backlogging. Eur. J. Oper. Res. 163 (2005) 776-783.

[16] J. Eliashberg and R. Steinberg, Marketing-production decisions in an industrial channel of distribution. Manage. Sci. 33 (1987) 981-1000.

[17] G.M. Erickson, A differential game model of the marketing-operations interface. Eur. J. Oper. Res. 211 (2011) $394-402$.

[18] G. Feichtinger and R. Hartl, Optimal pricing and production in an inventory model. Eur. J. Oper. Res. 19 (1985) $45-56$.

[19] L. Feng, Dynamic pricing, quality investment, and replenishment model for perishable items. Int. Trans. Oper. Res. 26 (2019) $1558-1575$.

[20] L. Feng, Y.L. Chan and L.E. Cárdenas-Barrón, Pricing and lot-sizing polices for perishable goods when the demand depends on selling price, displayed stocks, and expiration date. Int. J. Prod. Econ. 185 (2017) 11-20.

[21] L. Feng, J. Zhang and W. Tang, Dynamic joint pricing and production policy for perishable products. Int. Trans. Oper. Res. 25 (2018) 2031-2051.

[22] G. Fibich, A. Gavious and O. Lowengart, Explicit solutions of optimization models and differential games with nonsmooth (asymmetric) reference-price effects. Oper. Res. 51 (2003) 721-734.

[23] W. Fleming and R. Rishel, Deterministic and Stochastic Optimal Control. Springer, Berlin-Heidelberg (1975).

[24] A. Gabor and C. Granger, Price sensitivity of the consumer. J. Advert. Res. 4 (1964) 40-44.

[25] B. Giri, S. Pal, A. Goswami and K. Chaudhuri, An inventory model for deteriorating items with stock-dependent demand rate. Eur. J. Oper. Res. 95 (1996) 604-610.

[26] M.G. Güler, T. Bilgiç and R. Güllü, Joint pricing and inventory control for additive demand models with reference effects. Ann. Oper. Res. 226 (2015) 255-276.

[27] A. Herbon and K. Kogan, Time-dependent and independent control rules for coordinated production and pricing under demand uncertainty and finite planning horizons. Ann. Oper. Res. 223 (2014) 195-216.

[28] K.L. Hou and L.C. Lin, An EOQ model for deteriorating items with price-and stock-dependent selling rates under inflation and time value of money. Int. J. Syst. Sci. 37 (2006) 1131-1139.

[29] S. Jørgensen, P.M. Kort and G. Zaccour, Production, inventory, and pricing under cost and demand learning effects. Eur. J. Oper. Res. 117 (1999) 382-395.

[30] G. Kalyanaram and R.S. Winer, Empirical generalizations from reference price research. Mark. Sci. 14 (1995) $161-169$.

[31] K. Kogan, Production control under uncertainty: closed-loop versus open-loop approach. IIE Trans. 41 (2009) $905-915$.

[32] K. Kogan, B. Venturi and M. Shnaiderman, The effect of uncertainty on production-inventory policies with environmental considerations. IEEE Trans. 62 (2017) 4862-4868.

[33] P.K. Kopalle and R.S. Winer, A dynamic model of reference price and expected quality. Mark. Lett. 7 (1996) 41-52.

[34] Y.P. Lee and C.Y. Dye, An inventory model for deteriorating items under stock-dependent demand and controllable deterioration rate. Comput. Ind. Eng. 63 (2012) 474-482.

[35] R.I. Levin, C.P. McLaughlin, R.P. Lamone and J.F. Kottas, Productions/Operations Management: Contemporary Policy for Managing Operating Systems. McGraw-Hill, New York, NY (1972).

[36] S. Li, J. Zhang and W. Tang, Joint dynamic pricing and inventory control policy for a stochastic inventory system with perishable products. Int. J. Prod. Res. 53 (2015) 2937-2950.

[37] Y. Li, S. Zhang and J. Han, Dynamic pricing and periodic ordering for a stochastic inventory system with deteriorating items. Automatica 76 (2017) 200-213.

[38] L. Lu, J. Zhang and W. Tang, Optimal dynamic pricing and replenishment policy for perishable items with inventory-leveldependent demand. Int. J. Syst. Sci. 47 (2016) 1480-1494.

[39] B.N. Mandal and S. Phaujder, An inventory model for deteriorating items and stock-dependent consumption rate. J. Oper. Res. Soc. 40 (1989) 483-488.

[40] T. Mazumdar, S.P. Raj and I. Sinha, Reference price research: review and propositions. J. Mark. 69 (2005) $84-102$.

[41] U. Mishra, L.E. Cárdenas-Barrón, S. Tiwari, A.A. Shaikh and G. Trevino-Garza, An inventory model under price and stock dependent demand for controllable deterioration rate with shortages and preservation technology investment. Ann. Oper. Res. 254 (2017) 165-190.

[42] K.B. Monroe, Buyers' subjective perceptions of price. J. Mark. Res. 10 (1973) 70-80.

[43] G. Padmanabhan and P. Vrat, EOQ models for perishable items under stock dependent selling rate. Eur. J. Oper. Res. 86 (1995) 281-292.

[44] S. Pal, A. Goswami and K. Chaudhuri, A deterministic inventory model for deteriorating items with stock-dependent demand rate. Int. J. Prod. Econ. 32 (1993) 291-299.

[45] V. Pando, L.A. San-José, J. García-Laguna and J. Sicilia, Optimal lot-size policy for deteriorating items with stock-dependent demand considering profit maximization. Comput. Ind. Eng. 117 (2018) 81-93.

[46] D. Pekelman, Simultaneous price-production decisions. Oper. Res. 22 (1974) 788-794.

[47] I. Popescu and Y. Wu, Dynamic pricing strategies with reference effects. Oper. Res. 55 (2007) 413-429.

[48] K. Prasad and B. Mukherjee, Optimal inventory model under stock and time dependent demand for time varying deterioration rate with shortages. Ann. Oper. Res. 243 (2016) 323-334.

[49] S.P. Sethi and G.L. Thompson, Optimal Control Theory: Applications to Management Science and Economics. Kluwer, Dordrecht (2000).

[50] G. Sorger, Reference price formation and optimal pricing strategies, edited by G. Feichtinger. In: Vol. 3 of Optimal Control Theory and Economic Analysis. Elsevier (North Holland), Amsterdam (1988) 97-120. 
[51] G. Strang, Differential Equations and Linear Algebra. Wellesley-Cambridge Press, Wellesley, MA (2014).

[52] J.T. Teng and C.T. Chang, Economic production quantity models for deteriorating items with price-and stock-dependent demand. Comput. Oper. Res. 32 (2005) 297-308.

[53] H.M. Wee, A replenishment policy for items with a price-dependent demand and a varying rate of deterioration. Prod. Plan. Control 8 (1997) 494-499.

[54] H.B. Wolfe, A model for control of style merchandise. Ind. Manage. Rev. 9 (1968) 69-82.

[55] M. Xue, W. Tang and J. Zhang, Optimal dynamic pricing for deteriorating items with reference-price effects. Int. J. Syst. Sci. 47 (2016) 2022-2031.

[56] H.L. Yang, J.T. Teng and M.S. Chern, An inventory model under inflation for deteriorating items with stock-dependent consumption rate and partial backlogging shortages. Int. J. Prod. Econ. 123 (2010) 8-19.

[57] J. Zhang, W.K. Chiang and L. Liang, Strategic pricing with reference effects in a competitive supply chain. Omega 44 (2014) 126-135. 